\title{
WHITEHEAD GROUPS OF FINITE POLYHEDRA WITH NONPOSITIVE CURVATURE
}

\author{
BIZHONG HU
}

\section{Introduction}

Our results are on the Whitehead groups Wh $\Gamma=K_{1}(Z \Gamma) / H_{1} \Gamma \times Z_{2}$ of some groups $\Gamma$ relating to geometry. The strategy of using control topology plus geometry to study $\mathrm{Wh} \Gamma$ was previously used to prove $\mathrm{Wh} \pi_{1} M=0$ for closed flat manifolds $M$ by Farrell and Hsiang in [8]. The following more general result was proved using ideas that involved sphere bundles and geodesic flows.

1.1. Theorem (F. T. Farrell and L. E. Jones [11] in first order). Wh $\pi_{1} M$ $=0$ for any closed Riemannian manifold $M$ with nonpositive curvature.

In this paper we obtain a Whitehead group result concerning finite polyhedra of nonpositive curvature in two steps. The first step is to transform the problem to one about closed manifolds, by applying the idea of hyperbolization. In the second step we prove that the Whitehead group of a closed manifold with PL nonpositive curvature is zero as a result of improving some key ideas from [9], [11] and [15]. The meaning of a polyhedron with negative or nonpositive curvature was defined in [14] by Gromov to study hyperbolic groups. The result of this paper is as follows. It covers a major class of semihyperbolic groups.

1.2. Theorem. Wh $\Gamma=0, \Gamma=\pi_{1} K$ for any finite polyhedron $K$ with nonpositive curvature.

Note that this implies $K_{1}(Z \Gamma)=H_{1} \Gamma \times Z_{2}, \widetilde{K}_{0}(Z \Gamma)=0, K_{i}(Z \Gamma)=0$, $i \leq-1$. A previous result in this respect is the vanishing of Whitehead groups in the negative curvature case in [15]. One interest in extending that to the nonpositive curvature case is from the application of Theorem 1.2 to $p$-adic groups via their Euclidean buildings ([21]), which are the most interesting known examples of polyhedron nonpositive curvature structures. In particular there is 
1.3. Corollary. Let $Q_{p}$ be the field of p-adic numbers. For any torsionfree and cocompact discrete subgroup $\Gamma \subset \mathrm{SL}_{n}\left(Q_{p}\right), \mathrm{Wh} \Gamma=0$.

\section{From manifolds two polyhedra}

In this section we show

2.1. Lemma. If the Whitehead groups of closed manifolds with PL nonpositive curvature are zero, then the same is true for finite polyhedra.

It is hyperbolization, an idea due to Gromov [14] that allows us to see this. Because we only need to gain nonpositive rather than negative curvature, we will avoid the complicated and somewhat unclear strict hyperbolization needed in [15], by using here a weak but transparent hyperbolization. What we will define in 2.2 is the relative version of $[6,4 \mathrm{a}]$, which is like the untwisted version of the first hyperbolization of $[14,3.4]$.

Let $K$ be a finite simplicial complex, $A$ be a subcomplex. Do the following: Let $h K^{1}=K^{1}$; take $h K^{1} \times( \pm 1)=$ two copies of $h K^{1}$. If $\Delta^{2} \subset A$, then denote $h \Delta^{2}=\Delta^{2} \times( \pm 1)$. But if $\Delta^{2} \not \subset A$, then define $h \Delta^{2}=\partial \Delta^{2} \times[-1,1], h K^{2}=K^{1} \times( \pm 1) \cup$ all $h \Delta^{2}$, and so on. The end result $h(K, A)$, the hyperbolization of $K$ relative to $A$, is what we want to use. To make things clear we give

2.2. There is a unique construction $h$ with the following properties:

(1) For any finite simplicial complex $K^{n}$ and subcomplex $A, h(K, A)$ is a finite simplicial complex. If $L^{i}$ is a subcomplex of $K$, then

$$
h(L, L \cap A) \times( \pm 1)^{n-i} \subset h(K, A) .
$$

Here $h(L, L \cap A) \times( \pm 1)^{n-i}$ represents the disjoint union of $2^{n-i}$ copies of $h(L, L \cap A)$. Note that if $L$ is a set of vertices, then we should use $L \times( \pm 1)^{n-1}$ rather than $L \times( \pm 1)^{n}$, because the construction starts at dimension one, not zero.

Note. Here another interesting hyperbolization shows up if we start the construction at dimension zero.

(2) If $K^{i}, L^{j}$ and $A$ are subcomplexes of some finite simplicial complex $P^{n}, k=\operatorname{dim}(K \cap L)$, then

$$
\begin{gathered}
h(K, K \cap A) \times( \pm 1)^{n-i} \cup h(L, L \cap A) \times( \pm 1)^{n-j} \\
=h(K \cup L,(K \cup L) \cap A) \times( \pm 1)^{n-\max (i, j)}, \\
h(K, K \cap A) \times( \pm 1)^{n-i} \cap h(L, L \cap A) \times( \pm 1)^{n-j} \\
=h(K \cap L, K \cap L \cap A) \times( \pm 1)^{n-k} .
\end{gathered}
$$


(3) For any $A \subset \Delta^{1}, h\left(\Delta^{1}, A\right)=\Delta^{1}$. For $n \geq 2$

$$
\begin{aligned}
h\left(\Delta^{n}, \Delta^{n}\right) & =\Delta^{n} \times( \pm 1)^{n-1}, \\
h\left(\Delta^{n}, A\right) & =h\left(\partial \Delta^{n}, A\right) \times[-1,1], \quad \text { for any } A \subset \partial \Delta^{n} .
\end{aligned}
$$

Moreover, $\partial \Delta^{n} \subset \Delta^{n}$ induces $h\left(\partial \Delta^{n}, A\right) \times( \pm 1) \subset h\left(\partial \Delta^{n}, A\right) \times[-1,1]$.

Now assume that $K^{n}$ is a finite simplicial complex and $A$ is a subcomplex such that $\Delta^{i} \cap A$ is a simplex for any $\Delta^{i}$ in $K$.

2.3. Lemma. For any $A \subset L \subset K, h(L, A) \times( \pm 1)^{n-\operatorname{dim} L} \subset h(K, A)$ is $\pi_{1}$-injective. That means that the inclusion induces injections of fundamental groups at all connected components.

Proof. For a subcomplex $P$ and an integer $m$, we will denote $h P m=$ $h(P, P \cap A) \times( \pm 1)^{m-\operatorname{dim} P}$. Let $r$ be the number of simplices in $K$ that are not in $A$. First add $K^{1}$ to $L$. Note that $h L \cup K^{1} n$ is the union of $h L n$ and a one-dimensional complex, so Lemma 2.3 is true for them. Therefore we can assume that the dimensions of the simplices in $K$ but not in $L$ are $\geq 2$. Reduce the problem to one about $h P n \subset h\left(P \cup \Delta^{i}\right) n$, $A \subset P \subset P \cup \Delta^{i} \subset K$, where $i \geq 2, \Delta^{i} \not \subset P, \partial \Delta^{i} \subset P$. Write $\operatorname{dim} P=d$, $\max (d, i)=m$. Note that

$$
\begin{gathered}
h\left(P \cup \Delta^{i}, A\right)=h P m \cup h \Delta^{i} m, \\
h P m \cap h \Delta^{i} m=h \partial \Delta^{i} m, \\
h\left(\Delta^{i}, \Delta^{i} \cap A\right)=h\left(\partial \Delta^{i}, \partial \Delta^{i} \cap A\right) \times[-1,1] .
\end{gathered}
$$

To continue we need

2.3.1. Lemma. Let $X, Y$ and $X \cap Y=Z$ be compact polyhedra. If $Z \subset X$ and $Z \subset Y$ are $\pi_{1}$-injective, then $X \subset X \cup Y$ is $\pi_{1}$-injective.

Proof. Let $X_{0}$ be one connected component of $X, X_{0} \cap Z=Z_{0}$. Let $Y_{0}$ be the union of those components of $Y$ that have intersections with $Z_{0}$. Then the fundamental group of $X_{0}$ expands to that of $X_{0} \cup Y_{0}$ by generalized free products and HNN extensions. Let $Z_{1}$ be the union of components of $Z$ that are in $Y_{0}$ but not in $Z_{0}$. Let $X_{1}$ be the union of components of $X$ that intersect $Z_{1}$. Consider $X_{0} \cup Y_{0} \subset X_{0} \cup Y_{0} \cup X_{1}$. Note that the process terminates at a component of $X \cup Y$.

According to Lemma 2.3.1, and since $h\left(\partial \Delta^{i}, \partial \Delta^{i} \cap A\right) \times( \pm 1) \subset$ $h\left(\partial \Delta^{i}, \partial \Delta^{i} \cap A\right) \times[-1,1]$ is $\pi_{1}$-injective, the problem is reduced to $h \partial \Delta^{i} d$ $\subset h(P, A)$. Since $h A d \cap h \partial \Delta^{i} d=h A \cap \partial \Delta^{i} d, h A d=A \times( \pm 1)^{d-1}$, $h A \cap \partial \Delta^{i} d=\left(A \cap \partial \Delta^{i}\right) \times( \pm 1)^{d-1}$, and $\partial \Delta^{i} \cap A=\Delta^{i} \cap A=$ simplex, 
$h \partial \Delta^{i} d \subset h A \cup \partial \Delta^{i} d$ is $\pi_{1}$-injective. So the problem is reduced to $h A \cup$ $\partial \Delta^{i} d \subset h(P, A)$. The number of simplices in $P$ but not in $A$ is $\leq r-1$. This completes the proof of Lemma 2.3. Implicit in the proof is

2.4. Lemma. For $A \subset L \subset K, i \geq 2, \Delta^{i} \not \subset L, \partial \Delta^{i} \subset L, h\left(\partial \Delta^{i}, \partial \Delta^{i} \cap\right.$ $A) \times( \pm 1)^{\operatorname{dim} L-i+1} \subset h(L, A)$ is $\pi_{1}$-injective.

Let $K$ be a finite simplicial complex. Assume that each simplex of $K$ is a simplex with flat geometry such that all these geometric simplices can fit together. Now assume that the geometry of $K$ has nonpositive curvature, whose definition was made in $[14,4.2]$. Since a subdivision does not change this status, put $K$ as a subcomplex of a closed PL manifold $M$.

2.5. Lemma. Let $P$ be a finite simplicial complex and $A$ be a subcomplex with PL geometry. Then after a subdivision of $P$ that does not involve $A$, the PL geometry of $A$ can be extended over $P$.

Proof. If the relative dimension of $(P, A)$ is one, then assign any positive number to each $\Delta^{1} \not \subset A$. This gives a PL geometry to $P$. Assume $\operatorname{dim}(P, A)=n$ and Lemma 2.5 is true for $n-1$. Let $Q$ be a subdivision of $P^{n-1} \cup A$ such that the PL geometry of $A$ extends to one for $Q$. Consider any $\Delta^{n} \subset P, \Delta^{n} \not \subset A$. Note that a subdivision $\overline{\partial \Delta^{n}}$ of $\partial \Delta^{n}$ has PL geometry. We mention that for any geometric simplex $\Delta^{i}$, that is, a simplex positioned in $R^{i}, \partial \Delta^{i}$ bounds a unique sphere in $R^{i}$, and that if we use the center of the sphere, draw a straight line from the center, perpendicular to $R^{i}$, and pick a point from the line, then this point together with $\Delta^{i}$ spans a geometric simplex. Now we can verify that there is large $r>0$ such that for any simplex $\Delta^{i} \subset \overline{\partial \Delta^{n}}$, there is a unique geometric simplex (i.e., a simplex isometric to one in an Euclidean space) isometric to cone $C \Delta^{i}$ such that the length of the segment between the cone base point and any vertex of $\Delta^{i}$ is $r$. The union of these cones is the cone $C \overline{\partial \Delta^{n}}$ which can be identified as a subdivision of $\Delta^{n}$, giving it a PL geometry. This extends the PL geometry of $Q$ to $P$ and proves Lemma 2.5. q.e.d.

So we assume that the PL geometry of $K$ extends to one on $M$. Do a barycentric subdivision to make sure that $\Delta^{i} \cap K$ is a simplex for any $\Delta^{i}$ in $M$. It is known that $h(M, K)$ is a closed PL manifold and has nonpositive curvature ([6], [14, 3-4]). One way of proving $h(M, K)$ has nonpositive curvature is to show that the inclusion in Lemma 2.3 is totally geodesic so that everything in the following process of going from $K$ to $h(M, K)$ is totally geodesic (compare $[15,9]$ ).

2.6. Denote $\operatorname{dim} M=n$. For any subcomplex $P$ let $h P$ represent $h(P, P \cap K)$ in 2.6 . 


$$
h M=\left(K \cup M^{1}\right) \times( \pm 1)^{n-1} \bigcup_{\substack{i \geq 2 \\ \Delta^{i} \not \subset K}} h \Delta^{i} \times( \pm 1)^{n-i} .
$$

By Lemma 2.4 there is the following process of constructing the fundamental group of $h M$ from that of $K$. Recall that by convention $G_{1}{ }^{*}{ }_{H} G_{2}$ denotes a free product with amalgamation, and $G{ }^{*}{ }_{H} t$ denotes an HNN extension. Thus we have

$$
\begin{aligned}
& K \longrightarrow \pi_{1} K, \\
& K \cup M^{1} \longrightarrow \pi_{1} K * Z \cdots * Z, \\
& \cdot \\
& \cdot \\
& \cdot \\
& L=K \times( \pm 1)^{i-2} \cup h M^{i-1} \longrightarrow \pi_{1} L . \\
& \text { Take } \Delta^{i} \not \subset K, \\
& \widetilde{L}=L \times( \pm 1) \cup h \Delta^{i} \longrightarrow \pi_{1} L *_{\pi_{1} h \partial \Delta^{i}} \pi_{1} \\
& \text { Take another } \widetilde{\Delta}^{i} \not \subset K, \\
& \widetilde{L} \cup h \widetilde{\Delta}^{i} \longrightarrow \pi_{1} \widetilde{L}_{\pi_{1}} h \partial \tilde{\Delta}^{i} t \\
& \cdot \\
& \cdot \\
& \cdot \\
& K \times( \pm 1)^{i-1} \cup h M^{i}, \\
& \cdot \\
& \cdot \\
& \text {. } \\
& h M \longrightarrow \pi_{1} h M .
\end{aligned}
$$$$
\widetilde{L}=L \times( \pm 1) \cup h \Delta^{i} \longrightarrow \pi_{1} L *_{\pi_{1} h \partial \Delta^{i}} \pi_{1} L \text {. }
$$

Waldhausen's theorem in [23] says that the following two sequences are exact:

$$
\begin{aligned}
& \mathrm{Wh}(H) \longrightarrow \mathrm{Wh}\left(G_{1}\right) \oplus \mathrm{Wh}\left(G_{2}\right) \longrightarrow W h\left(G_{1} *_{H} G_{2}\right), \\
& \mathrm{Wh}(H) \longrightarrow \mathrm{Wh}(G) \longrightarrow \mathrm{Wh}\left(G *_{H} t\right)
\end{aligned}
$$

Since $h \partial \Delta^{i}$ are closed manifolds with PL nonpositive curvature, their Whitehead groups are zero by assumption. Then we get $\mathrm{Wh}\left(\pi_{1} K\right) \subset$ $\mathrm{Wh}\left(\pi_{1} h M\right)$ which is zero again by assumption. This proves Lemma 2.1 .

\section{Proof of the manifold case}

3.1. In $\S 3$ we will prove

3.1.1. Theorem. Wh $\pi_{1} M=0$ for any closed PL manifold $M$ with nonpositive curvature. 
$M$ having nonpositive curvature means that each simplex in $M$ is assigned a flat geometry of certain size and that any link of $M$ is larger than or the same as a standard sphere [14, 4.2]. The example of plane $R^{2}$ can be thought of as the composition of angles at the origin with total sum $\sum=2 \pi$. When one inserts more angles, say letting the sum become $\sum=4 \pi$, then the metric on $R^{2}$ is just the pullback, by $z^{2}: R^{2} \rightarrow R^{2}$, of $d s^{2}=d x^{2}+d y^{2}$, which is $d \tilde{s}^{2}=4\left(x^{2}+y^{2}\right) d s^{2}$, a Riemannian metric with singularity. So $M$ is like having a metric with various singularities, which may be the background of the following geodesic singularity (a geodesic going into different directions), which is our main concern:

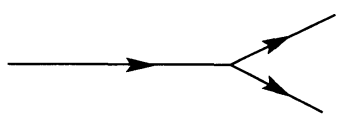

FIGURE 1

Three things are used to overcome this difficulty. They are the geodesic flow $G$ which is the collection of parametrized geodesics, the sphere bundle $R$ which is the collection of geodesic rays, and bundle $S_{T}$ which is the collection of segments of length $T$.

3.1.2. Note that closed manifolds of almost nonpositive curvature (limits of Riemannian manifolds of $K \leq 0$, see [13]) are covered by Theorem 3.1.1 because their universal covers satisfy the property that the distance functions of two geodesics are convex. In fact they are far less complicated here in that there is no geodesic singularity. So Whitehead groups of the fundamental groups of them must also vanish.

3.2. The spaces $G, R$ and $S_{T}$.

Note that in $\S 3$ we always assume $M^{n}$ to be a closed PL manifold with curvature $\leq 0$, and $X$ its universal cover. First recall that the geodesic flow of $M$ is $G(M)=$ all local isometries $R \rightarrow M$ \}, and if $\tau \in R$, $\alpha(t) \in G(M)$, then let $(\tau \alpha)(t)=\alpha(\tau+t)$. There is a metric on $G(M)$ that comes from one on $G(X)$ by defining distance in $G(M)$ to be the minimum of distances between elements in the inverse images in $G(X)$. The metric on $G(X)$ is

$$
d(\alpha, \beta)=\int_{-\infty}^{+\infty} d[\alpha(t), \beta(t)] \cdot e^{-|t|} d t .
$$

3.2.1. Lemma. For geodesics $\alpha(t), \beta(t)$ in $M$ and liftings $\tilde{\alpha}(t), \tilde{\beta}(t)$ in $X, d(\alpha, \beta) \leq d(\tilde{\alpha}, \tilde{\beta})$. 
The sphere bundle of $M$ is $R(M)=$ local isometries $[0,+\infty) \rightarrow$ $M$ \}, which is a fiber bundle over $M$ with fiber the ideal boundary of $X$. Denote the ideal boundary by $\partial X$, which is homeomorphic to $S^{n-1}$.

3.2.2. Theorem (see $[15, \S 4])$. The canonical map $G(M) \rightarrow R(M)$ can be approximated by homeomorphisms.

For $T>0$, let $S_{T}(M)$ be the set of all parametrized geodesic segments of length $T$ in $M$. Its topology is from $S_{T}(X)$, in which two elements are closed if and only if they are closed pointwise.

3.2.3. Lemma. $\quad S_{T}(M) \rightarrow M$ is a fiber bundle.

This assertion is equivalent to that the homeomorphism approximations of $S_{b}(x) \rightarrow S_{a}(x)$ for $a<b$ can depend continuously on $x$ in $X$. Davis and Januszkiewicz in [6] proved that $S_{b}(x) \rightarrow S_{a}(x)$ can be approximated by homeomorphisms, where $S_{r}(x)$ is the sphere of radius $r$ with center $x$ in $X$. In particular, any $S_{r}(x)$ is homeomorphic to $S^{n-1}$.

Let $A$ and $B$ be locally compact, separable and metric spaces, and $f: A \rightarrow B$ proper and surjective. $f$ being completely regular means that, for each $y_{0}$ in $B$ and $\varepsilon>0$, there is a neighborhood $U$ for $y_{0}$ such that: for each $y$ in $U$, there is a homeomorphism $h: f^{-1}(y) \rightarrow f^{-1}\left(y_{0}\right)$ which is $\varepsilon$-closed to $\mathrm{Id}_{A}$.

3.2.4. Theorem (Dyer-Harmstrom). If $f: A \rightarrow B$ is completely regular, $B$ is locally finite-dimensional, and the point-inverses $f^{-1}(y)$ have locally contractible homeomorphism groups, then $f$ is fiber bundle.

See [7, Theorem 3 and the "Note" at the bottom of p. 109].

Proof of Lemma 3.2.3. We will just show that $S_{T}(X)$ is a fiber bundle. In view of the above criterion one should show $S_{T}(X) \rightarrow X$ to be completely regular. Note that $\operatorname{Homeo}\left(S^{n-1}\right)$ being locally contractible is known as a result of the Cernavskii theorem. Give $S_{T}(X)$ the following metric:

$$
d(\alpha, \beta)=d[\alpha(0), \beta(0)]+d[\alpha(T), \beta(T)] .
$$

Assume that $\varepsilon>0, x, y$ are in $X, d(x, y) \leq \varepsilon / 4$. Choose a homeomorphism $f_{x}: \partial X \rightarrow S_{T}(x)$ that is $\varepsilon / 4$-closed to the canonical map. A similar $f_{y}: \partial X \rightarrow S_{T}(y)$ is chosen. Consider Figure 2 (next page), where $\tilde{u}=\left(f_{x}^{-1} u\right)(T), \tilde{v}=\left(f_{y}^{-1} v\right)(T)$.

$$
\begin{aligned}
d(x, y)+d(u, v) & \leq d(x, y)+d(u, \tilde{u})+d(\tilde{u}, \tilde{v})+d(\tilde{v}, v) \\
& \leq d(x, y)+d(u, \tilde{u})+d(x, y)+d(\tilde{v}, v) \\
& \leq 4 \cdot \varepsilon / 4=\varepsilon
\end{aligned}
$$




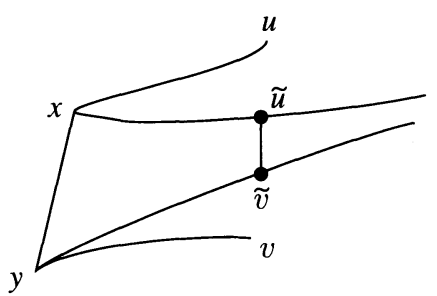

FIGURE 2

where $d(\tilde{u}, \tilde{v}) \leq d(x, y)$ because the distance function of two asymptotic geodesics is a decreasing one. This means that $f_{y} f_{x}^{-1}: S_{T}(x) \rightarrow S_{T}(y)$ is $\varepsilon$-closed to Id in $S_{T}(X)$.

3.2.5. Corollary. There is a continuous family of maps $h_{t}: R(M) \rightarrow$ $S_{T}(M), t \in[0,1)$, such that $h_{t}, t \in[0,1)$, are bundle equivalences and $h_{1}$ is the canonical map.

Proof. For any ray $\alpha(t), t \in[0,+\infty)$, one gets segment $\alpha(t), t \in$ $[0, T]$. This is the canonical map, which is cell-like by argument similar to that of Theorem 3.2.2. Since $S_{T}(M)$ is indeed a manifold by Lemma 3.2 .3 , [20] can imply $h_{t}$ (see [20, Complement to Theorem A] and the remark that follows. A weaker statement that follows from the complement is enough for us).

3.3. Technical estimates.

Let $\alpha(t), 0 \leq t \leq 1$, be a curve in $M$, and $\gamma(s), 0 \leq s<+\infty$, be a geodesic ray with $\gamma(0)=\alpha(0)$. Assume that the diameter of $\alpha$ is $\leq d$, $T>0$. Lift $\alpha$ and $\gamma$ to $X$ to be $\tilde{\alpha}$ and $\tilde{\gamma}$ such that $\tilde{\alpha}(0)=\tilde{\gamma}(0)$. For each $t$ in $[0,1]$, draw the geodesic segment from $\tilde{\alpha}(t)$ to $\tilde{\gamma}(T+d)$. Since the length of this segment is $\geq T+d-d=T$, a smaller segment of length $T$, denoted $\tilde{\alpha}(t) * \tilde{\gamma}$, is available. Map it down to $M$. The result, written as $\alpha(t) * \gamma$, is independent of ways of lifting and is a curve in $S_{T}(M)$.

Assume that $W$ is $h$-cobordism over $M, p_{t}, q_{t}, 0 \leq t \leq 1,: W \times$ $[0,1] \rightarrow W$ are defomrations of $W$ to $M$ and to another boundary. The lifting of $W$ to $R(M)$ to

$$
\widehat{W}=R(M) \times_{M} W=\left\{(\gamma, x) \in R(M) \times W: \gamma(0)=p_{1}(x)\right\}
$$

Assume that the maximum of the diameters of the curves (called associated curves of the $h$-cobordism) $p_{1} p_{t} x, p_{1} q_{t} x, x \in W$, is $d$, which by definition is the diameter of $W$. Take $T>0$. Use $d$ and $T$ to obtain, for any $(\gamma, x)$ in $R(M) \times W$ with $\gamma(0)=p_{1}(x)$, a curve $p_{1} p_{t} x * \gamma$ in 
$S_{T}(M)$. Take the $h_{t}$ of Corollary 3.2.5. Consider

$$
\begin{gathered}
\left\{\left(h_{0}^{-1} h_{t} \gamma, x\right), 0 \leq t \leq 1\right\} \cup\left\{\left(h_{0}^{-1} p_{1} p_{t} x * \gamma, p_{t} x\right), 0 \leq t \leq 1\right\} \\
\cup\left\{\left(h_{0}^{-1} h_{1-t} \gamma, p_{1} x\right), 0 \leq t \leq 1\right\}
\end{gathered}
$$

where the notation $\cup$ means the three curves are wedged together. This is a curve in $\widehat{W}$. Let $i: R(M) \rightarrow \widehat{W}$ be the inclusion, and $j: \widehat{W} \rightarrow R(M)$ be $(\gamma, x) \rightarrow \gamma$. Then $j i=\mathrm{Id}$; Id is homotopic to $i j:(\gamma, x) \rightarrow\left(\gamma, p_{1} x\right)$ via the collection of the above expressed curves. Therefore the associated curves in $R(M)$ are

$$
\begin{aligned}
\left(h_{0}^{-1} h_{t} \gamma, 0 \leq t \leq 1\right) & \cup\left(h_{0}^{-1}\left[p_{1} p_{t} x * \gamma\right], 0 \leq t \leq 1\right) \\
& \cup\left(h_{0}^{-1} h_{1-t} \gamma, 0 \leq t \leq 1\right), \quad(\gamma, x) \text { in } R(M) \times_{M} W .
\end{aligned}
$$

Consider the other boundary in the same way. One sees

3.3.1. Lemma. Let $W$ be h-cobordism over $M$ with diameter $d, T>$ 0 . Then there are homotopies (weak deformations) of $\widehat{W}=R(M) \times_{M} W$ to its boundaries, and a homeomorphism $h_{0}: R(M) \rightarrow S_{T}(M)$ such that the associated curves of $h_{0}(\widehat{W})$ in $S_{T}(M)$ are arbitrarily closed to the following curves:

$$
p_{1} p_{t} x * \gamma, 0 \leq t \leq 1, p_{1} q_{t} x * \gamma, 0 \leq t \leq 1:(\gamma, x) \in R(M) \times_{M} W .
$$

We now prepare to change the above curves. For any $\varepsilon>0$, take $h_{0}$, and choose a homomorphism $g_{0}: G(M) \rightarrow S_{T}(M)$ which is very closed to the canonical map denoted by $f$. Consider $g_{0}^{-1} h_{0}(\widehat{W})$ of which any associated curve, restricted from $G(M)$ to $S_{T}(M)$, can be $\varepsilon$-closed to a curve of the form $\alpha(t) * \gamma$, where $\alpha \subset M$, $\operatorname{diam}(\alpha) \leq d, \gamma$ is in $R(M)$, and $\gamma(0)=\alpha(0)$. Give $G_{T}(X)$ the metric $d(\alpha, \beta)=d[\alpha(0), \beta(0)]+$ $d[\alpha(T), \beta(T)]$, which is invariant under isometries and induces a metric on $S_{T}(M)$. Consider any curve $V$ in $G(M)$ such that $d(f V, \alpha * \gamma) \leq \varepsilon$. There must be a a lifting $\widetilde{f V}$ of $f V$ to $S_{T}(X)$ such that $d(\widetilde{f V}, \tilde{\alpha} * \tilde{\gamma})=$ $d(f V, \alpha * \gamma) \leq \varepsilon . V$ and $\widetilde{f V}$ determine $\widetilde{V}$ which is a lifting of $V$ and $f \widetilde{V}=\widetilde{f V}, d(f \tilde{V}, \tilde{\alpha} * \tilde{\gamma}) \leq \varepsilon$. Adding Lemma 3.2.1 into consideration, we can simply look at the following situation.

Given a fixed $d>0$, any $\varepsilon>0, T>0$, and a collection $\Sigma(\varepsilon, T)$ of curves in $G(X)$ such that for any one of its curves, there is a geodesic segment $\gamma[0, T+d]$ in $X$ such that for any point on the curve, expressed as geodesic $\alpha(t), t \in R$, there is the triangle in $X$ shown in Figure 3 (next page) such that $d[\alpha(0), \bar{\gamma}(0)] \leq \varepsilon, d[\alpha(T), \bar{\gamma}(T)] \leq \varepsilon$. The purpose is to make $\Sigma$ closed to leaves of $G(X)$. We remind the reader that for 
510

BIZHONG MU

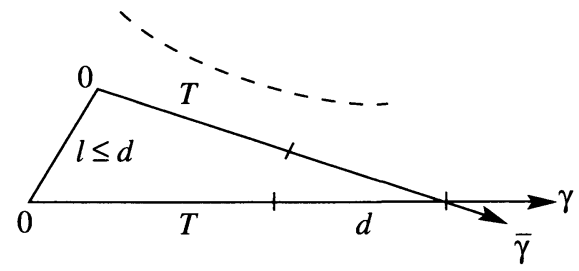

FIGURE 3

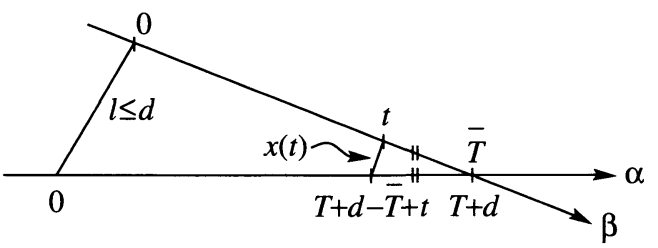

FIGURE 4

any $\omega \in G(X)$, there is leaf $\mathbb{R} \omega \subset G(X)$. The following three points are needed.

(1) For any triangle $(l, a, b)$ in $X$, with $l \leq d,|a-b| \leq l \leq d$. By the metric formula in $\S 3.2$, a segment of length $d$ in $X$ means a segment of length $2 d$ in $G(X)$.

(2) Consider two geodesics $\alpha(t)$ and $\beta(t)$ in $X$ as shown in Figure 4. Since $X$ has curvature $\leq 0$,

$$
x(t)=d(\beta(t), \alpha(T+d-\bar{T}+t)) \leq \frac{T+d-t}{T} 2 d .
$$

For $t$ in $[(1-2 \varepsilon) T, T]$,

$$
x(t) \leq 2 d^{2} / T+4 d \varepsilon
$$

Let $\tau=(1-\varepsilon) T$. We estimate

$$
\begin{aligned}
d[\tau \beta,(T+d-\bar{T}+\tau) \alpha] & =\int_{-\infty}^{+\infty} d[\beta(\tau+t), \alpha(T+d-\bar{T}+\tau+t)] e^{-|t|} d t \\
& =\int_{-\infty}^{-\varepsilon T}+\int_{-\varepsilon T}^{\varepsilon T}+\int_{\varepsilon T}^{+\infty}, \\
\int_{-\varepsilon T}^{\varepsilon T} & =\int_{-\varepsilon T}^{\varepsilon T} x(\tau+t) e^{-|t|} d t \\
& \leq 4 d^{2} / T+8 d \varepsilon .
\end{aligned}
$$




$$
\begin{array}{rl}
\int_{-\infty}^{-\varepsilon T} & d[\beta(\tau+t), \alpha(T+d-\bar{T}+\tau+t)] e^{-|t|} d t \\
& \leq \int_{-\infty}^{-\varepsilon T}\left[2(-\varepsilon T-t)+\frac{2 d^{2}}{T}+4 d \varepsilon\right] e^{-|t|} d t \\
& \leq 2 e^{-\varepsilon T}+\frac{2 d^{2}}{T}+4 d \varepsilon .
\end{array}
$$

The same is true for the integration from $\varepsilon T$ to $+\infty$. So

$$
d[\tau \beta,(T+d-\bar{T}+\tau) \alpha] \leq 4 e^{-\varepsilon T}+8 d^{2} / T+16 d \varepsilon .
$$

(3) If there are two geodesics $\alpha(t)$ and $\beta(t)$ in $X$ such that $d[\alpha(0), \beta(0)] \leq \varepsilon, d[\alpha(T), \beta(T)] \leq \varepsilon, \tau=(1-\varepsilon) T$, then $d(\tau \alpha, \tau \beta)$ $\leq 4 e^{-\tau}+4 \varepsilon$.

These three points together imply that $\tau \cdot \Sigma(\varepsilon, T)$ is foliate controlled (a terminology of Farrell-Jones) by the following bound. Note that for a class of curves in a one-dimensional foliation we say it is $(u, v)$-controlled, or its diameter is $\leq(u, v)$, if any curve in the class is in a $v$-neighborhood of some leaf segment whose length is $\leq u$. If $T=1 / \varepsilon^{2}$ and $\varepsilon \rightarrow 0$, then the second coordinate of

$$
\left(2 d, 4 e^{-\varepsilon T}+\frac{8 d^{2}}{T}+16 d \varepsilon+4 e^{-(1-\varepsilon) T}+4 \varepsilon\right)
$$

goes to zero. This gives

3.3.2. Proposition. Let $W$ be $h$-cobordism over $M$ with diameter $d$, and $\widehat{W}$ be the lifting of $W$ to $R(M)$. Then for any $\delta>0$ there is a homeomorphism $g=\tau g_{0}^{-1} h_{0}: R(M) \rightarrow G(M)$ such that $g(\widehat{W})$ is $(2 d, \delta)$ controlled.

3.4. Proof of Theorem 3.1.1.

We now prove Theorem 3.1.1, i.e., Wh $\pi_{1} M=0$. It is okay to consider $M \times S^{1}$ instead of $M$ because $\mathrm{Wh} \pi_{1} M \subset \mathrm{Wh}\left(\pi_{1} M \times Z\right)$. Orient $S^{1}$. Then there is the natural decomposition $S_{T}\left(M \times S^{1}\right)=S_{T}^{+} \cup S_{T}^{0} \cup S_{T}^{-}$, which comes from a decomposition of $S_{T}(X \times R)$, because if we take $\gamma$ in $S_{T}(X)$, then $\left(S_{T}(\gamma \times R)\right)_{0}$, the union of which for all $\gamma$ being $S_{T}(X \times R)$, has the natural decomposition shown in Figure 5 (next page). Apparently there are similar decompositions $G\left(M \times S^{1}\right)=G^{+} \cup G^{0} \cup G^{-}, R\left(M \times S^{1}\right)=$ $R^{+} \cup R^{0} \cup R^{-}$.

Any element of $\mathrm{Wh}\left(\pi_{1} M \times Z\right)$ is the Whitehead torsion $\tau(W)$ of an $h$-cobordism $W$ over $M \times S^{1}$. Lift $W$ to $\widehat{W}$ over $R\left(M \times S^{1}\right)$. Then $\widehat{W}=\widehat{W}^{+} \cup \widehat{W}^{0} \cup \widehat{W}^{-}$, and $\widehat{W}^{+} \cup \widehat{W}^{0}$ is $h$-cobordism over $R^{+} \cup R^{0}$, which 
BIZHONG HU

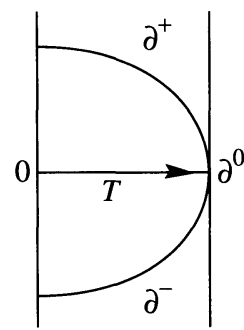

$$
\left(S_{T}(\gamma \times \mathbb{R})\right)_{0}=\partial^{+} \cup \partial^{0} \cup \partial^{-}
$$

Figure 5

is a fiber bundle over $M \times S^{1}$, with disc $E^{n+1}$ as fiber. So $\tau\left(\widehat{W}^{+} \cup \widehat{W}^{0}\right)=$ $\tau(W)$, and we consider $\widehat{W}^{+} \cup \widehat{W}^{0}$.

To apply Proposition 3.3.2 to change this $h$-cobordism, choose $h_{t}$ and $g_{0}$ there to respect decompositions. A problem is that the homotopies of $\widehat{W}^{+} \cup \widehat{W}^{0}$ constructed at the beginning of $\S 3.3$ may go out of $\widehat{W}^{+} \cup \widehat{W}^{0}$. Take neighborhood $\partial^{0} \cup \partial^{-1 / 2}$ for $\partial^{0} \subset \partial^{0} \cup \partial^{-}$. Then the obvious retraction $\partial^{0} \cup \partial^{-1 / 2} \rightarrow \partial^{0}$ induces a map $J: S_{T}^{+} \cup S_{T}^{0} \cup S_{T}^{-1 / 2} \rightarrow S_{T}^{+} \cup S_{T}^{0}$. Now change each $p_{1} p_{t} x * \gamma, \gamma \in R^{+} \cup R^{0}, \gamma(0)=p_{1} x$, to $J\left(p_{1} p_{t} x * \gamma\right)$. This gives us a correct homotopy of $\widehat{W}^{+} \cup \widehat{W}^{0}$. But then we expect $J\left(p_{1} p_{t} x * \gamma\right)$ to be very closed to $p_{1} p_{t} x * \gamma$. This is true if the projection of $p_{1} p_{t} x$ from $M \times S^{1}$ to $S^{1}$ is small. To prove this, take large $k$ in $Z$, consider Id $\times z^{k}: M \times S^{1} \rightarrow M \times S^{1}$, and substitute $W$ by $W_{k}=\left(\operatorname{Id} \times z^{k}\right)^{*} W$. If $\tau\left(W_{k}\right)=0$ is proved, then $k \cdot \tau(W)=0$. Take another large $l$ in $Z$, such that $(k, l)=1$. As $k \cdot \tau(W)=l \cdot \tau(W)=0, \tau(W)=0$. It is true that we have an alternate approach due to a referee: Choose the $h$-cobordism $W$ over $M \times S^{1}$ to represent an element in the image of the inclusion $\mathrm{Wh} \pi_{1} M \rightarrow \mathrm{Wh}\left(\pi_{1} M \times Z\right)$, i.e., choose $W$ so that it is a product over $M \times\left(S^{1}\right.$-very small arc $)$. Then those curves in $S^{1}$ will automatically be very small.

So apply Proposition 3.3.2 to get a homeomorphism $g: R^{+} \cup R^{0} \rightarrow$ $G^{+} \cup G^{0}$ such that $g\left(\widehat{W}^{+} \cup \widehat{W}^{0}\right)$ is $(2 d, \delta)$-controlled, where $\delta$ can be arbitrarily small. Now it is better to add a trivial $h$-cobordism over $G^{0} U$ $G^{-}$(see Lemma 3.8 of [9]) so that we need only consider an $h$-cobordism $\widetilde{W}$ over $G$, that is $(4 d, \delta)$-controlled, where $\delta$ can be arbitrarily small. Now we turn to

3.4.1. Theorem. Assume that $m \geq 5$, and $G^{m}$ is a manifold with 1-dimensional foliation. Also assume that $A \subset G$ is compact such that any leaf intersecting $A$ has length $>l$. Then for any $\varepsilon>0$ there is $\delta>0$ such that the following is true: For any h-cobordism $H$ over $G$ with 
$\operatorname{diam}(H) \leq(l, \delta)$, there is handlebody structure for $H$ such that there is no handle over $A$ and that the diameter of the handlebody structure is. $\leq(C(m) l, \varepsilon)$.

This is an adjustment of $[15,7.6]$ to the language used by Quinn ([18] or [19]) to consider handlebody structures of $h$-cobordisms directly without having to mention the concept of products which will not be enough later.

In our case let $l=4 d$. Let $G_{4 d}$ be the union of all closed orbits in $G$ with periods $\leq 4 d$. Then $\widetilde{W}$ as well as its handlebody structure are $(D, \varepsilon)$-controlled and all handles are over a neighborhood of $G_{4 d}$, where $D=C(2 n-1) 4 d$ depends on $d$ and $n$ only, $\varepsilon$ can be arbitrarily small and the neighborhood can be arbitrarily close to $G_{4 d}$. We now want to apply the thin $h$-corbordism to $G_{4 d}$ because the $h$-cobordism is very close to the circles in $G_{4 d}$. One should notice that $G_{4 d}$ is not fibered by $S^{1}$ although [11] shows it can be filtered into a stratification of fiber bundles. But we will see that the local situation of $G_{4 d}$ is still within the ability of [18].

3.4.2. Definition. Let $N$ be a closed manifold with PL nonpositive curvature.

(1) If $\alpha(t), t \in R$, is a closed geodesic with period (i.e., minimum period) $u$, and $k \geq 1$ is an integer, then we can have a map $S^{1}(k u) \rightarrow$ $[0, k u] / 0=k u \stackrel{\alpha}{\rightarrow} N$. Call this map, together with the orientation and the length of $S^{1}(k u)$ but dropping the reference point, a $k$-fold circle from $\alpha$. The period of this circle means $u$ not $k u$.

(2) If $S^{1}(u) \times[a, b] \rightarrow N$ is a totally geodesic immersion such that the circles at $(a, b)$ are all one-fold, then call it a primitive move from the one-fold version of the circle at $a$ to the one-fold version of the circle at $b$. Call $u$ the period of the primitive move, and $b-a$ its perpendicular distance. A move is a combination of several primitive moves. The perpendicular distance of a move means the sum of those of the primitivies. A down move is a combination of primitive moves such that the period of any primitive move is equal to that of its beginning circle.

3.4.3. Lemma. Let $N$ be as above.

(1) Let $Y \rightarrow Y / \Gamma=N$ be the universal cover of $N$. In this paragraph $S^{1}(T)$ denotes the circle of length $T>0$ with orientation and base point $0 \in S^{1}(T)$. Let $f: S^{1}(A) \rightarrow N, g: S^{1}(B) \rightarrow N$ be totally goedesic immersions. Recall that a standard application of the fact that the distance function of any two geodesics in $Y$ is convex implies that, if $f$ and $g$ are homotopic, then $A=B=T$, there exist a totally geodesic embedding $R \times[a, b] \subset Y$ where $R \times a$ lifts $f$ and $R \times b$ lifts $g$, and $h \in \Gamma$, 
$h(t, s)=(t+T, s),(t, s) \in R \times[a, b]$, and up to isometries of $\Gamma$ the flat band is uniquely determined by the homotopy class of the path from $f(0)$ to $g(0)$ with fixed endpoints.

(2) Assume that $l>0$, and $\alpha \in G=G(N)$ such that $R \alpha$ is closed and of length $\leq l$. Let $U$ be a regular neighborhood of $R \alpha$ in $G$. Then there exists $\lambda>0$ such that for any $\beta \in G, d(\beta, R \alpha) \leq \lambda$ implies $\beta \in U$. Moreover, if there exists $\mu>0$ such that for any $\beta \in G$, length $(R \beta) \leq l$, and $d(\beta, R \alpha) \leq \mu$, then $d(\tau \beta, R \alpha) \leq \lambda$, for any $\tau \in R$.

Proof. If $\mu$ does not exist, then there should be a sequence $\beta_{i} \in G$, such that length $\left(R \beta_{i}\right) \leq l, d\left(\beta_{i}, a_{i} \alpha\right) \leq 1 / i, d\left(\tau_{i} \beta_{i}, R \alpha\right)>\lambda$. We can suppose that $0 \leq a_{i}, \tau_{i} \leq l$, and therefore that $a_{i} \rightarrow a, \tau_{i} \rightarrow \tau$. Thus

$$
d\left(\beta_{i}, a \alpha\right) \leq d\left(\beta_{i}, a_{i} \alpha\right)+d\left(a_{i} \alpha, a \alpha\right) \leq 1 / i+2\left|a_{i}-a\right|,
$$

which implies that $\beta_{i} \rightarrow a \alpha$ so that $\tau \beta_{i} \rightarrow \tau a \alpha$, which $d\left(\tau_{i} \beta_{i}, \tau \beta_{i}\right) \leq$ $2\left|\tau_{i}-\tau\right|$. Hence $\tau_{i} \beta_{i} \rightarrow \tau a \alpha$, which is impossible.

(3) Let $l>0$, and $\alpha \in G$ such that length $(R \alpha)=T \leq l$ and $d>0$. Then there exists $\varepsilon>0$ such that for any $\beta \in G$, length $(R \beta)=A \leq l$, $d(\beta, \alpha) \leq \varepsilon$, there is a totally geodesic immersion $S^{1}(A) \times[a, b] \rightarrow N$ going from the 1-fold circle of $\beta$ to a certain fold circle of $\alpha$ with $|a-b| \leq$ $d$.

Proof. Choose a regular neighborhood $U$ of $R \alpha$ in $G$. By (2) we know that when $\varepsilon>0$ is small enough and $\beta$ is as mentioned above, the whole $R \beta \subset U$. Since $U$ is homotopic to $R \alpha$, there must be a homotopy of $R \beta$ to $(R \alpha)^{k}$, where $k \geq 1$ is some integer. Let $f: S^{1}(A) \rightarrow N$ be the 1 -fold circle of $\beta$, and $g: S^{1}(k T) \rightarrow N$ be the $k$-fold circle of $\alpha$. Then $G(N) \rightarrow N$ induces a homotopy from $f$ to $g$. By (1) we get a totally geodesic immersion $S^{1}(A) \times[a, b] \rightarrow N$ going from $f$ to $g$. If we choose $U$ to be thin in advance, we can make sure that $|a-b| \leq d$.

(4) Let $l>0$, and $\alpha \in G$ such that length $(R \alpha) \leq l$. Then there exists $\delta>0$ such that for any totally geodesic immersion $S^{1}(T) \times[a, b] \rightarrow N$ from a circle of some $\beta$ to a circle of $\alpha, T \leq l,|a-b| \leq \delta$, and it must be a down move. Any other move from $\beta$ to $\alpha$ of periods $\leq l$ has perpendicular distance $>|a-b|$.

Proof. Find $d>0$ such that for any $x, y \in N, d(x, y) \leq d$, there is a unique geodesic segment connecting $x$ and $y$. By (3) there is $\varepsilon>0$. Let $\delta=\min (d, \varepsilon / 2)$. Details are left for the reader.

(5) Any totally geodesic immersion $S^{1}(T) \times[a, b] \rightarrow N$ is a move. q.e.d. of 3.4.3.

Let $N$ be as above, $l>0$, and $G_{l}$ be the set of all closed orbits of $G=G(N)$ with lengths $\leq l$. Let $B$ be the set of all 1 -fold circles with 
periods $\leq l$. From the topological point of view we should divide $B$ into disconnected subsets using the equivalence relation that two elements in $B$ are equivalent if and only if between them there is a move consisting of primitive moves of periods $\leq l$. In a component define the distance between two elements to be the lower bound of the perpendicular distances of all the moves between them. This gives a metric to $B$ : Suppose $\alpha, \beta \in$ $G_{l}, S^{1}(T) \times[a, b] \rightarrow N$ is a totally geodesic immersion going from a circle of $\alpha$ to one of $\beta$. Then there is a pair of points respectively in $R \alpha$ and $R \beta$ with distance $\leq 2|a-b|$. If $|a-b|$ can be arbitrarily small, then $R \alpha=R \beta$. Other details are omitted.

Consider the canonical map $f: G_{l} \rightarrow B$, which is continuous by (3) and (5) above. For any element $e \in B$, there is $r_{0}>0$ such that for any $0 \leq r \leq r_{0}$, the closed ball $E_{r}$ of radius $r$ shrinks to $e$ while $f^{-1}\left(E_{r}\right)$ shrinks to $f^{-1}(e)$ which is homeomorphic to $S^{1}$.

Note that notation in this paragraph and Theorem 3.4.4 below are independent of the preceding notation. Let $M^{n}$ be a closed manifold, $n \geq 5$, $X$ be compact subset in $M$, and $W$ be $h$-cobordism over $M$ with deformations $p_{t}$ and $q_{t}, 0 \leq t \leq 1$, to $\partial_{-} W=M$ and to $\partial_{+} W$. Recall that for an $x \in W$ it has two associated curves $p_{1} p_{t} x$ and $p_{1} q_{t} x$. For $\varepsilon>0$, $X^{\varepsilon}$ denotes the set of points that are $\varepsilon$-closed to some points in $X$. Let $\delta$ be $>0, k \geq 0$ be integer. We say that $W$ is $(X, \delta, k)$-controlled if there is

$$
0=\delta_{0}<\cdots \delta_{k}<\delta_{k+1}=\delta
$$

such that the associated curves of $p_{1}^{-1}\left(X^{\delta_{i}}\right)$ are in $X^{\delta_{i+1}}, 0 \leq i \leq k$. A handlebody structure of $W$ is over $X^{\varepsilon}$ if all handles are inside $p_{1}^{-1}\left(X^{\varepsilon}\right)$. Let $W_{-1}$ be the collar part of the handlebody structure with homeomorphism $h_{t}: M \times[0,1] \rightarrow W_{-1}, h_{0}=\mathrm{Id}_{M}$. Note that a handlebody structure for $W$ means a sequence $W_{-1} \subset W_{0} \subset \cdots \subset W_{n+1}$, where $W_{i}$ is obtained from $W_{i-1}$ by adding $i$-handles, $0 \leq i \leq n+1$. Recall that for an $x \in M$ its associated curve is $p_{1} h_{t} x . W_{-1}$ is $(X, \delta, k)$-controlled if the associated curves of $X^{\delta_{i}}$ are in $X^{\delta i+1}$. Assume that $U$ is a neighborhood of $X$ in $M, u$ is a retraction of $U$ to $X, B$ is a compact metric space and $f: X \rightarrow B$ is a continuous map. $W$ is $\varepsilon$-controlled at $B$ if the diameters of the images under $f u$ of the associated curves of $W$ that are inside $U$ are $\leq \varepsilon . W_{-1}$ being $\varepsilon$-controlled at $B$ is understood in a similar way.

3.4.4. Theorem. Assume that $X$ is locally contractible, $B$ is locally 1-connected, and for any point $\mathrm{pt} \in B$ and any sufficiently small $r>0$ the counter image $f^{-1}(E)$ of the closed $r$-ball $E$ of the point is homotopic 
to $f^{-1}(p t)$. If $\mathrm{Wh}\left(\pi_{1}\left(f^{-1} p t\right) \times Z^{i}\right)=0, i \geq 0$, then there are $\varepsilon_{0}>0$, $\delta_{0}>0$, and $k_{0}$ that depends only on $n$, such that for any $\varepsilon \leq \varepsilon_{0}, \delta \leq \delta_{0}$ and $k \geq k_{0}, W$ is trivial.

This is a slight extension of 2.7 in [18] (also see [3]) by treating a subset $X$ of a manifold rather than the whole manifold $M$ itself. The above arrangements are to ensure that controlled handle eliminations can be carried out near $X$. The reader can now see that $[18, \S 6]$ works for Lemma 3.4.3. $X$ being locally contractible makes sure that there are always neighborhood retractions.

Return to the situation following Theorem 3.4.1. $\widetilde{W}$ is $(D, \varepsilon)$-controlled. When $\varepsilon$ is small enough, the above theorem applies to $\widetilde{W} \supset G \supset$ $G_{4 d} \stackrel{f}{\rightarrow} B$, in particular, to $\mathrm{Wh}\left(\pi_{1} S^{1} \times Z^{i}\right)=0, i \geq 0$, so that $\widetilde{W}$ is trivial. This proves Theorem 3.1.1. Note that it should be possible to obtain the same conclusion by arranging $f$ into a stratification of fiber bundles by proving [11, Theorem 2.4] in our PL setting. We might use this approach when proving Theorem 1.2 for higher Whitehead groups.

\section{Appendix to $\S 2$}

4.1. Theorem. Let $i$ be a fixed integer.

(1) If $\mathrm{Wh}_{i} \pi_{1} M=0$ for any closed manifold $M$ with $P L$ curvature $\leq 0$ then $\mathrm{Wh}_{i} \pi_{1} K=0$ for any finite simplicial complex $K$ with curvature $\leq 0$.

(2) If $\mathrm{Wh}_{i} \pi_{1} M=0$ for any closed aspherical manifold $M$ then $\mathrm{Wh}_{i} \pi_{1} K$ $=0$ for any finite aspherical simplical complex $K$.

By the main theorem of [24] for any $G_{1}{ }^{*}{ }_{H} G_{2}$ there is an exact sequence $\mathrm{Nil}_{i} \oplus \mathrm{Wh}_{i}(H) \stackrel{j}{\rightarrow} \mathrm{Wh}_{i}\left(G_{1}\right) \oplus \mathrm{Wh}_{i}\left(G_{2}\right) \rightarrow \mathrm{Wh}_{i}\left(G_{1} *_{H} G_{2}\right), j\left(\mathrm{Nil}_{i}\right)=0$. So $\mathrm{Wh}_{i}(H) \rightarrow \mathrm{Wh}_{i}\left(G_{1}\right) \oplus \mathrm{Wh}_{i}\left(G_{2}\right) \rightarrow \mathrm{Wh}_{i}\left(G_{1} *_{H} G_{2}\right)$ is exact, and for any $G *_{H} t, \mathrm{Wh}_{i}(H) \rightarrow \mathrm{Wh}_{i}(G) \rightarrow \mathrm{Wh}_{i}\left(G *_{H} t\right)$ is exact. Other details of applying $\S 2$ to Theorem 4.1 are left to the reader. There is no extra problem in an analogue of Theorem 4.1 for $L$-theory, but we expect that more than such an analogue will be needed and so do not state it here.

\section{References}

[1] J. Alonzo \& M. Bridson, Semihyperbolic groups, preprint.

[2] K. Brown, Buildings, Springer, New York, 1989.

[3] T. A. Chapman, Controlled boundary and h-cobordism theorems, Trans. Amer. Math. Soc. 280 (1983) 73-95.

[4] T. A. Chapman \& S. Ferry, Approximating homotopy equivalences by homeomorphism, Amer. J. Math. 101 (1979) 583-607. 
[5] R. Charney \& M. Davis, Singular metrics of nonpositive curvature on branched covers of riemannian manifolds, preprint, Ohio State University.

[6] M. Davis \& T. Januszkiewicz, Hyperbolization of polyhedra, J. Differential Geometry, to appear.

[7] E. Dyer \& M. E. Harmstrom, Completely regular mappings, Fund. Math. 45 (1957) 103-118.

[8] F. T. Farrell \& W. C. Hsiang, The topological Euclidean space form problem, Invent. Math. 45 (1978) 181-192.

[9] F. T. Farrell \& L. E. Jones, K-theory and dynamics. I, Ann. of Math (2) 124 (1986) 531-569.

[10] __, Algebraic K-theory of discrete subgroups of Lie groups, Proc. Nat. Acad. Sci. U.S.A. 84 (1987) 3095-3096.

[11] __ Stable pseudoisotopy spaces of compact non-positively curved manifolds, J. Differential Geometry 34 (1991) 769-834.

[12] __ Foliated control without radius of injectivity restrictions, Topology 30 (1991) 117142.

[13] K. Fukaya \& T. Yamaguchi, Almost nonpositively curved manifolds, to appear.

[14] M. Gromov, Hyperbolic groups, Essays in Group Theory (S. M. Gersten, ed.), Math. Soc. Res. Inst Publ. Vol. 8, 1987, 175-264.

[15] B. Hu. A PL geometric study of algebraic K-theory, Trans. Amer. Math. Soc., to appear.

[16] G. Moussong, Hyperbolic Coxeter groups, Thesis, Ohio State Univ., 1988.

[17] A. J. Nicas \& C. W. Stark, Whitehead groups of certain hyperbolic manifolds, Math. Proc. Cambridge Philos. Soc. 95 (1984) 299-308.

[18] F. Quinn, Ends of maps. I, Ann. of Math. (2) 110 (1979) 275-331.

[19] _ _ Ends of maps. II, Invent. Math. 68 (1982) 353-424.

[20] L. C. Siebenmann, Approximating cellular maps by homeomorphisms, Topology 11 (1973) 271-294.

[21] J. L. Tits, On buildings and their applications, Proc. Internat. Congr. Math. (Vancouver, 1974), 209-221.

[22] E. B. Vinberg, Discrete linear groups generated by reflections, Math. USSR Izv. 5 (1971) 1083-1119.

[23] F. Waldhausen, Whitehead groups of generalized free products, Lecture Notes in Math., Vol. 342, Springer, 1973, 155-179.

[24] _ _ Algebraic K-theory of generalized free products, Ann. of Math. (2) 108 (1978) 135-256.

Max-Planck-Institut fur Mathematik, Bonn 
